Healthcare Settings. Draft document, http://www.cdc.gov/HAI/ organisms/norovirus.html.

\section{QuantiFERON-TB Testing for Latent Tuberculosis Infection in Low-Prevalence Countries: Making the Most of an Imperfect Process}

To the Editor-The commentaries of Gandra et al ${ }^{1,2}$ and Joshi et $\mathrm{a}^{3,4}$ reflect the experiences that we have also had with QuantiFERON-TB Gold (QFT-G) ${ }^{5}$ and then with QuantiFERON-TB Gold In Tube (QFT-GIT). ${ }^{6}$ During initial testing of selected new hospital employees as well as subsequent annual testing for tuberculosis, conversions and reversions have occurred with surprising frequency among those employees with high negative values and those with low positive values. Because of our concerns about broadly replacing the timetested, if itself imperfect, tuberculin skin test (TST), we have restricted the use of interferon gamma release assay (IGRA) testing to hospital employees who are TST-positive, BCG vaccine recipients. This itself eliminates one of the concerns cited by Joshi et $\mathrm{al}_{,}{ }^{4}$ that of deciding what to do about those with positive IGRA and negative TST results.

Initially using QFT-G, we found that only $13.5 \%(29 / 215)$ of our TST-positive, BCG recipient new employees tested positive. ${ }^{7}$ This increased to $30.2 \%(38 / 126)^{6}$ when we introduced QFT-GIT and now hovers between $23 \%$ and $24 \%$ (70/ 302 ), as would be anticipated with a reportedly more sensitive test. ${ }^{8}$ However, it is important to recognize that by virtue of adding IGRA testing to the TST, we have reduced the percentage of those to whom we offered treatment for latent tuberculosis infection (LTBI) by more than $70 \%$. This is important in addressing a multinational and urban employee population such as ours, with nearly one-quarter of our new employees testing tuberculin positive.

In addition to or in place of repeat testing, ${ }^{3}$ one can also emphasize clinical judgment more decisively in determining whether to propose treatment for those with borderline positive IGRA results. Thus, factors such as suggestive chest $\mathrm{x}$ ray findings, relative youth, recent immigration from a tuberculosis-endemic area, and coincident illnesses or treatment programs wherein $\mathrm{TB}$ is either more frequent or more threatening serve as inducements, while advanced age and slight liver function abnormalities act as constraints. Additionally, a decisively positive IGRA test result or a confirmed more modest response can reinforce both the practitioner and the patient in advancing LTBI treatment plans. Thus, this test process, while still imperfect in the context that we use it, offers distinct advantages over TST testing alone.

\section{ACKNOWLEDGMENTS}

Potential conflicts of interest. All authors report no conflicts of interest relevant to this article.

\section{William J. Schneider, MD; ${ }^{1,2}$ Arthur E. Brown, MD; ${ }^{1,2}$ Cynthia Eisenstein, $\mathbf{R N}^{1}$}

Affiliations: 1. Employee Health and Wellness Services, Memorial SloanKettering Cancer Center, New York, New York; 2. Weill Cornell Medical College, New York, New York.

Address correspondence to William J. Schneider, MD, Employee Health and Wellness Services, Memorial Sloan-Kettering Cancer Center, 222 East 70th Street, New York, NY 10021 (schneidw@mskcc.org).

Infect Control Hosp Epidemiol 2011;32(10):1055-1055

(C) 2011 by The Society for Healthcare Epidemiology of America. All rights reserved. 0899-823X/2011/3210-0020\$15.00. DOI: $10.1086 / 662022$

\section{REFERENCES}

1. Gandra S, Scott WS, Somaraju V, Wang H, Wilton S, Feigenbaum M. Questionable effectiveness of the QuantiFERON test (Cellestis) as a screening tool in healthcare workers. Infect Control Hosp Epidemiol 2010;31:1279-1285.

2. Gandra S, Scott WS, Somaraju V. Reply to Joshi et al. Infect Control Hosp Epidemiol 2011;32:518-519.

3. Joshi M, Monson T, Woods G. Practical experience with the QFTGIT assay for LTBI annual testing among US heath-care workers in a large tertiary setting. Chest 2010;138:746A.

4. Joshi M, Monson T, Woods G. QuantiFERON-TB test for annual screening of healthcare workers: not yet ready for prime time in low-prevalence countries. Infect Control Hosp Epidemiol 2011;32: 518.

5. Schneider WJ, Eisenstein C, Brown AE, Patel PH. Higher than expected annual QuantiFERON-Gold (QFT-G) "conversion" rate suggests a need for redefining reactivity criteria in healthcare workers (HCWs). 47th annual meeting of the Infectious Diseases Society of America, 29 October-1 November 2009; Philadelphia.

6. Schneider WJ, Brown AE, Eisenstein C. Successive comparative observations of test results using QuantiFERON-Gold (QFT-G) and QuantiFERON-Gold In Tube (QFT-GIT): implications in a health worker setting. 48th annual meeting of the Infectious Diseases Society of America, 23 October 2010; Vancouver. Abstract 4947; poster LB-24.

7. Brown AE, Eisenstein C, Schneider WJ, Kiehn TE, Glickman M. Results of QuantiFERON-TB Gold (QFT-G) testing of employees in a healthcare setting. 45th annual meeting of the Infectious Diseases Society of America, 4-7 October 2007; San Diego, CA. Abstract LB-12.

8. Harada N, Higuchi K, Yoshiyama T, et al. Comparison of the sensitivity and specificity of two whole blood interferon-gamma assays for $M$. tuberculosis infection. J Infect 2008;56:348-353. 